\title{
Diagnostic Problems of Occult HBV Infection
}

ISSN: 2637-7632

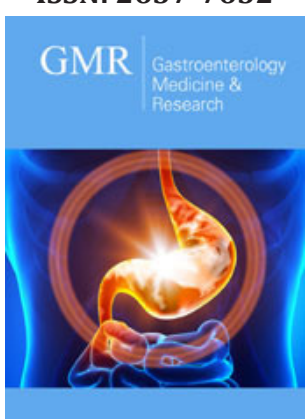

*Corresponding author: Morozov IA ,Chumakov Federal Scientific Center for research and development of immuneand-biological products of Russian Academy of Sciences, Russia

Submission: 眥 March 11, 2020

Published: 㭗 March 18, 2020

Volume 4 - Issue 4

How to cite this article: Morozov IA Ilchenko L Yu, Fedorov IG and Tkachenko PE. Diagnostic Problems of Occult HBV Infection. Gastro Med Res. 4(4). GMR.000594. 2020.

DOI: 10.31031/GMR.2020.04.000594

Copyright@ Morozov IA, This article is distributed under the terms of the Creative Commons Attribution 4.0 International License, which permits unrestricted use and redistribution provided that the original author and source are credited.

\author{
Morozov IA ${ }^{1}$, Ilchenko L Yu ${ }^{1,2}$, Fedorov IG $^{2,3}$ and Tkachenko PE \\ ${ }^{1}$ Chumakov Federal Scientific Center for research and development of immune-and-biological \\ products of Russian Academy of Sciences, Russia \\ ${ }^{2}$ Pirogov Russian National Research Medical University, Russia \\ ${ }^{3}$ State Clinical hospital named after VM Buyanov, Russia \\ ${ }^{4}$ Sechenov First Moscow State Medical University, Russia
}

\begin{abstract}
In this report we present current data on prevalence, clinical significance and methods of diagnostics of occult hepatitis B (OHB). Mechanisms of occult hepatitis B formation are reviewed, among which the most important is co-infection with other hepatotropic viruses. Diagnostic significance of anti-HBcAg antibodies is shown. An algorithm of occult hepatitis B detection developed by the authors involves two stages: detection of viral markers in serum and liver tissue. Attention is drawn to the role of morphologic methods including histochemistry, immunohistochemistry and electron microscopy in verification of HBV infection and direct visualization of virions in cytoplasm of hepatocytes.
\end{abstract}

\section{Introduction}

\section{Historical reference}

Until it was believed that HBV-infection can proceed in one of three forms: acute hepatitis B (HBV) characterized symptoms of acute liver lesions and intoxication (with or without jaundice); chronic hepatitis $\mathrm{B}$ (CHB) characterized prolonged inflammatory liver damage and persistence surface $\mathrm{HBV}$ antigen (HBsAg) in the blood of patients with no clinically significant hepatitis. However, case of acute hepatitis B containing antibodies to the capsid protein the HBV (anti-HBcore) in the absence of HBsAg and antibodies to it (anti-HBs) was registered in the recipient after the transfusion of blood in 1978. It was further shown that the virus DNA can be detected in the blood serum and liver tissue of patients in whom serum HBsAg was not detected by available methods. This phenomenon is called latent HBV -infection [1]. It should be noted, that HBsAg is still the main and sometimes the only serum marker of HBV, determined in accordance with the sanitary and epidemiological rules Russia to eliminate HBV -infection not only in blood donors, but also in patients with suspected CHB. Because of this, latent HBV infection is a serious problem not only for hepatologists, but also for blood transfusion centers in countries where, in addition to washed blood cells, transfusion also uses plasma or even whole blood for transfusion. Despite the fact that the latent HBV infection was discovered almost a quarter century ago, this phenomenon, like the whole problem of viral hepatitis, has long remained in the shadow of another infection, namely the human immunodeficiency virus (HIV), which was considered a disease of the century. However, at present, viral hepatitis in terms of the complexity of solving the problem came out on top among socially significant infectious diseases.

\section{The concept of «latent HBV -infection»}

In 2008 the term "latent HBV -infection" was defined as "the presence of HBV DNA in the liver of patients (regardless of the presence of HBV DNA in serum) in the blood serum of whom HBsAg is not determined by available methods at a conference of experts Taormina organized by the European Association for the Study of Liver (EASL) [2] . The definition of latent HBV infection indicates that the diagnostic search limited to the HBsAg study is ineffective, and infected individuals will join the group of patients with hepatitis of unknown etiology. According Hollinger \& Sood [3], approximately $20 \%$ of patient sera with the presence of latent HBV -infection not determined no serological markers of HBV -infection other than HBV DNA. Torbenson \& Thomas [4] found that $50 \%$ of sera contain antibodies to the capsid 
protein HBV -HBcore Ag (with or without anti-HBs); 35\% of sera contain antibodies to HBsAg (with or without anti-HBc). They believed that, based on the described data, HBV can be divided into a seropositive and seronegative variant, in which anti-HBcore and anti-HBs are not detected in the blood serum of patients. In patients with the latter option, serum HBV DNA levels are generally minimal. Seropositive patients can be further divided into two groups depending on the presence of anti-HBs in the serum. HBV DNA levels are highest in patients who are positive for anti-HBc and negative for anti-HBs. Such patients are most likely to transmit the infection to others. Patients positive both anti-HBc and anti-HBs, is generally determined by the average level of the HBV DNA.

Most often, HBV is recorded in the first days after HBV infection, in the so -called "serological window", when HBsAg has not yet been determined in the blood serum of patients. Anyway, latent $\mathrm{HBV}$-infection can also be detected in patients positive for the anti$\mathrm{HBc}$ and having chronic hepatitis B with HBsAg below detection level that is often associated with the appearance of anti-HBs. Such a pattern is observed in $0.7-1.3 \%$ of cases per year and is associated with an older age and the presence of anti-HBe in serum [5]. It should be borne in mind that the above data were obtained more than 15 years ago when significantly fewer sensitive methods for the detection of serum antigens and antibodies were used. According to the requirements of leading international organizations working in the field of health (WHO), the sensitivity assessment of tests used for screening donated blood should be assessed, first of all, using seroconversion panels, as well as panels of native samples obtained from patients containing various mutant forms HBsAg.

In the last decade, the use of new highly sensitive molecular biological methods has led to an increase in the detection rate of HBV and a better understanding of its biological and clinical features. This condition has been described in healthy blood donors, patients with chronic liver disease (CHD), and patients with hepatocellular carcinoma (HCC). However, several aspects of latent HBV-infection have not yet been studied, including the clinical significance of latent HBV-infection in relation to the risk of transmission of infection, its reactivation and the possibility of progression of CHD.

\section{Pathogenetic causes}

CHB-infection has phase course and during the fifth phase (final) is HBsAg -negative phase, which is characterized by the absence of HBsAg in serum of blood and the presence of anti-HBc with or without defined anti -HBs. This phase is sometimes called "latent (latent) HBV infection". Patients at this stage have normal ALT values and usually, but not always, undetectable in the blood serum HBV DNA, which is often found in liver tissue. Latent HBV infection is characterized by the presence of HBV DNA (i.e. episomal HBV covalently closed circular DNA [cccDNA]) in the liver tissue and / or HBV DNA in serum in HBsAg negative patients. The reasons for the persistence of small amounts of HBV DNA in the absence of a detectable level of HBsAg are still uncertain. About There is general agreement that in this phenomenon are important factors, related both to the virus, and with the owner [1]. Zerbini et al. [6] studied and specific for HBV T-cell response in patients with latent HBV -infection serum which were present or absent anti -HBc IgG. In patients positive for anti - $\mathrm{HBc}$ IgG, a typical protective immunity developed, which indicates a possible resolution of the infection. In contrast, a specific T-cell response to HBV was not observed in patients negative for anti-HBc IgG, which indicates the absence of the formation of a mature protective immune response at low levels of virus replication. These results confirm the difference in the mechanisms of control of viral replication in seropositive and seronegative patients with HBV.

In addition to the influence of factors associated with the host's immune response, a low level of viral replication may be due to the presence of defective viral particles, as well as mutations in the regions responsible for transcriptional control, leading to replication inefficiency [1]. The effect of cytokines on viral transcription leads to a decrease in the level of replication and expression of HBsAg, as well as an undetectable level of HBV DNA in blood serum if it is present in the liver tissue. It was also shown that HBsAg is involved in the regulation of hepatocyte release of new virions and non-infectious subviral particles. Enhancement of L -protein (large protein HBsAg) accelerates the formation of viral particles and their secretion, and inhibits the release of noninfectious subviral particles, whose number in the liver to 5-6 times higher number of full virions. In contrast, a low level of L-protein leads to the reverse development of DNA-containing nucleocapsids and an increase in the number of secreted subviral particles. The absence of HBsAg in the blood can be caused not only by the above-mentioned effect of proinflammatory cytokines on viral transcription, leading to a decrease in the level of replication and expression of HBsAg and HBV DNA, but also by a number of other reasons that can act simultaneously: the formation of $m$ immune complexes between HBsAg and anti-HBs undetectable by current methods [7]; the occurrence of mutations in the "a" -determinant of the $\mathrm{S}$ gene, leading to amino acid substitutions in the main hydrophilic loop of HBsAg and the inability to detect HBsAg using the currently used methods of its detection, as well as co-infection with hepatitis D viruses, HCV, HIV, leading to a decrease in the level synthesis of HBsAg and HBV replication [8]. It is possible that such first effect of causing hepatitis family Anelloviridae (TTV, Torque teno midi virus -TTMDV).

H. Ohto et al. established low level infection Anelloviridae early and follow present a rapid increase in prevalence (22\% -after 6 months and 33\% -after 2 years), which is consistent with the dependent age give TTV non-parenteral routes. Et and observations strongly suggest that TTV acquisition main factor in children is the increase in age interaction with infected material [9]. Despite the fact that the infection of HBV in the world of children is reduced due to effective vaccination, especially among infants, etc. on our opinion, the incidence of chronic viral hepatitis co-infected with various hepatotropic viruses in recent years has increased substantially. It is known that co-infection of HBV in patients with chronic hepatitis $\mathrm{C}$ (CHC) can significantly affect the course of the 
disease, the level of viral load, and the effectiveness of treatment. In patients infected with both HBV and HCV, compared with HBV monoinfection, HBsAg appears later and is detected in serum for less time, the HBV DNA concentration is lower, as well as the level of alanine aminotransferase (ALT) [10]. It was previously shown that core protein HCV inhibits the replication of HBV and the expression of its genes [11]. This observation has recently been called into question. Bellecave et al. [12] showed the possibility of replication of both viruses in vitro on the HUH7 cell line. It can be assumed that the effect of coinfection on HBV replication is associated with the action of indirect mechanisms such as innate and acquired host immunity [10,13]. Thus, absence reliable information on the characteristics of flow CHC in co-infection HBV, flowing in a latent form, $\mathrm{n}$ e allows to develop recommendations diagnosis and conducting such patients. It is possible that co-infection with other, not even hepatotropic viruses, can also affect not only the clinical manifestations of the disease, but also the pathogenetic mechanisms of the development of pathology.

\section{Prevalence of latent HBV infection}

The prevalence of occult HBV infection varies by region, specificity, and sensitivity of research methods. According to data of different publications the prevalence of latent HBV -infection varies in different treatment groups [1]. Its occurrence ranges from $0 \%$ to $2.4 \%$ among HBsAg-negative, anti -HBc-positive (but anti -HBs + -) blood donors in Western countries (such as in the USA, where only $5 \%$ of the population is lifetime met with HBV ), up to $6 \%$-in similar groups of donors living in endemic areas, where $70-90 \%$ of the population met with HBV [14]. However, most researchers around the world noted that latent (occult) HBV infection occurs on average in $20 \%$ of patients with chronic hepatitis B and depends on the endemicity of the region [1]. So, in the general population in the countries of Southeast Asia -7.5-16\% [15]. Earlier, in 1999, Cacciola et al. [16] was on a comparison of the prevalence of latent HBV HIV among patients with CHC. For this purpose, 200 HBsAg-negative patients with HCV infection were examined, while 100 anti -HBc were detected in the blood serum, and the rest did not have any serological markers of HBV infection. However, serum HBV DNA was detected not only in 46 patients with anti-HBc (46\%), but also in 20 patients without anti -HBc (20\%). In a recent study in Italy, HBV was detected in $16.3 \%$ of 98 patients without any clinical or biochemical signs of liver disease [17]. Most patients with GBS was anti-HBc $(62,5 \%)$. In another study in Japan, Marusawa et al. [18], evidence of the presence of HBV was shown in 13 of 14 healthy liver donors seropositive for anti-HBc and anti-HBs; at the same time, HBV DNA was not detected in any patient with "isolated" anti-HBs. It remains unclear whether HBV patients are a potential source of HBV infection, whether their course of other diseases is changing, and whether they are at increased risk of developing complications associated with HBV. Fashion lation Mr. B on the North American marmots (a woodchuck the hepatitis Bed and-WHB) showed the possibility of transmission of the virus from mother to fetus, which may indicate the possibility of vertical transmission in humans [1921]. The one-year-old marmots that had undergone WHB infection and developed "protective" antibodies had small amounts of the virus in the liver cells and peripheral blood mononuclear cells. In animals, inflammation in the liver tissue was also observed, which ultimately led to the development of HCC. Controversial data are available regarding the clinical significance of the presence of small amounts of HBV DNA in the body of immunocompetent patients who have undergone acute hepatitis B and those without clinical or biochemical manifestations of liver disease. It is generally believed that patients who have undergone acute hepatitis B have no clinical signs of liver disease in addition to small amounts of HBV DNA in serum, peripheral blood mononuclear cells and/or liver tissue, which can be determined decades later [11]. Along with this, the clinical problem of reactivation of HBV in connection with the use of immunosuppressive therapy [22], drugs with direct antiviral effect in the treatment of chronic hepatitis C [23], etc. has become recognized in recent years. Among high-risk groups for reactivation of HBV American Gastroenterological Association (AGA) isolates HBsAg-negative/anti-HBcore-positive patients taking prednisolone 10-20 mg or more per day for 4 weeks; patients receiving derivatives of anthracycline. The appearance of reactivation of HBV often leads to the development of active hepatitis and increases the risk of deaths [22]. The AGA recommends monitoring HBV DNA and the use of antiviral drugs with a high genetic barrier for the prevention of reactivation of $\mathrm{HBV}$ in patients at risk.

\section{Diagnosis and its algorithm for latent HBV infection}

A review of major publications on the issue of latent HBV infection in the last decade confirms the contradictory information on the prevalence, clinical significance and methods of diagnosis of this form of chronic hepatitis. This is especially true for the diagnostic value of anti -HB core. Their appearance in the blood occurs very quickly after infection with HBV and, most often, precedes HBs Ag. It is believed that of anti-HBc can be found in each who had contact with the HBV, and that they are present in different phases of the disease even after the elimination of the virus.

That is why the EASL expert group , which met in Taormina [2], considered anti -HBc to be an "imperfect "marker of HBV infection, but all the same recommended its use as a surrogate one when the HBV DNA test is not available to detect seropositive individuals with HBV , for example, in the case of blood donation and organ, as well as in patients undergoing their immunosuppressive therapy. According to the opinion of the Hollinger \& Sood [3], the best way to diagnose HBV is to detect HBV DNA in the liver tissue. However, they believe that liver biopsy is not always possible, and methods for detecting HBV DNA in liver tissue are not standardized. Madejon et al. [24] that the length of time between the preparation of a biopsy and its freezing is a critical factor for the conservation of nucleic acids and their subsequent detection. This period of time should not exceed 3 minutes. At the same time, in most cases of HBV, a biopsy is justified, since in addition to the possibility of determining HBV DNA in the liver tissue, it is possible to morphologically study it using a special immunocytochemical (ICC) and electron microscopic (EM) study and etiology of the disease. However, from 
the moment discovery of the phenomenon of hidden currents CHB morphological studies were not conducted, as evidenced by all of us cited publication. of the hidden course of chronic hepatitis B, morphological studies have not been conducted, as evidenced by all publications cited by us. Given these shortcomings in dealing with latent HBV infection us, based on the suggestions of experts EASL, developed algorithm, which consists of two research phases: serological and tissue [25], which was used in our studies. The first stage began with the determination of serum HBsAg and further related to enzyme-linked immunosorbent assay (ELISA) and polymerase chain reaction (PCR) of all serum samples of HBsAgnegative patients with chronic liver disease (CHD).

\section{Serological steps for detecting latent HVB infection}

The most sensitive immunobiological methods for verification of HBV infection were used in the work. In the blood serum, HBsAg was determined by a test system with a sensitivity of $0.01 \mathrm{ng} \mathrm{HBsAg}$ per $1 \mathrm{ml}$ of the test sample (DS ELISA HBsAg 0.01, produced by the NPO Diagnostic Systems, Nizhny Novgorod). Detection of HBsAg indicated the presence of HBV infection.

Anti-HBcore was examined by the "DS ANTI-HBc" test system of the same manufacturer. Detection of anti-HBcore allowed a preliminary diagnosis of HBV infection.

Detection of HBV DNA in blood serum was carried out by twostage PCR (sensitivity -150 copies or less $/ \mathrm{ml}$ ) with primers that overlap the core, $\mathrm{S}$ and $\mathrm{P}$ regions of the DNA molecule of this virus. Positive HBV DNA confirmed the diagnosis of HBV infection. When a negative PCR considered two possible options: carriage of HBsAg and false positive anti-HBc.

\section{Tissue steps for detecting latent HBV infection}

Biopsy material with the appropriate preparation was used for histopathological, ICC, EM studies. Successively applied the following methods:

A. Morphological examination of liver tissue to assess the activity and stage of the disease.

B. Histological staining with orcein on HBsAg according to Shikata.

\section{IHC detection of HBsAg.}

D. PCR to detect HBV DNA in liver tissue.

E. EM study of a liver biopsy specimen to detect virions, their species affiliation with HBV (ICC HBsAg), and changes specific for HBV infection.

Positive results from any special techniques serve 1 basis for definitive diagnosis "HBV -infection" if the presence of virions proved or formulated as "latent form CHB".

Among the surveyed our 168 patients with CKD of anti -the HBcore anti-HBcore was found in 60 (35.7\%). Of these 60 patients were excluded those with stablished diagnosis CHB (HBsAg+) and selected 35 patients whose serum was absent and HBsAg and
HBV DNA with diagnoses: CHC $(n=12)$, chronic alcoholic hepatitis $(n=9)$ non-alcoholic steatohepatitis $(n=4)$, mixed hepatitis (nonalcoholic hepatitis in patients with chronic alcohol intoxication, $n=2)$ and chronic hepatitis of unknown etiology $(n=6)$. One patient was suspected occult HB and one -diagnosed monoinfection TTV. In most patients of the study group, the clinical diagnosis suggested the presence of intracellular lipid infiltration in the liver tissue. However, only 4 out of 35 patients was detected globular moderate infiltration of triglycerides, and from 4 -weakly expressed small droplet form, i.e. most of the examined patients lacked one of the main pathognomonic signs of alcoholic or nonalcoholic steatohepatitis, and the clinical diagnosis was apparently made on the basis of ultrasound data as well as anamnestic information. In order to detect latent HBV infection, an IHC of biopsy material was performed with positive and negative controls. At IHC with monoclonal antibodies to HBsAg and HBcAg in all biopsies it was detected HBsAg. However, the response to the HBcore of $\mathrm{Ag}$ in the majority of cases in patients with monoinfected her HBV was mild, indicating a low replicative activity and chronic, not Active the during the slaughtering Levan (Figure 1a \& 1b). An EM study of biopsy material in all patients revealed the presence of HBV virions in the cytoplasm of hepatocytes (Figure 2a). Viral particles are spherical formations of moderate electron density, with scalloped edges and mild glycocalyx. The average diameter of the virions was $49.281 \pm 2.637 \mathrm{~nm}$, which is slightly larger than with negative contrast (42 nm) with uranyl acetate or tungsten phosphoric acid, which overlap the scalloped edge and glycocalyx of the virions. The type of virions in the cytoplasm of hepatocytes resembles the "scattered pea effect"; moreover, the virions do not touch each other. The affiliation of these virions to HBV is confirmed by EM ICC with monoclonal antibodies to HBsAg and the use of Protein A -Colloidal Gold complex to visualize the antigen-antibody reaction (Figure 2b).

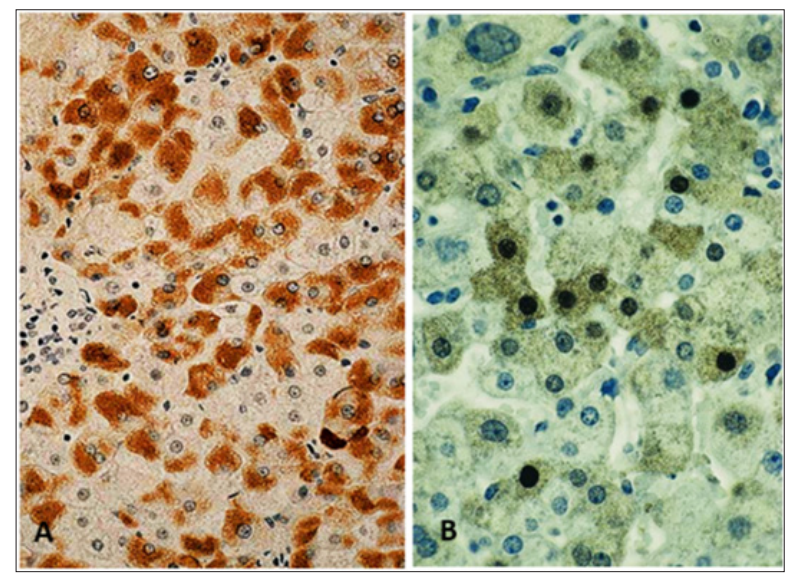

Figure 1: IHC of the patient's liver in case of chronic HBV monoinfection.

(a) HBsAg in hepatocytes, in the accumulation phase. x 200;

(b) A small amount of HBcore Ag with HB with minimal activity and poor replication of the virus. $x$ 400. 


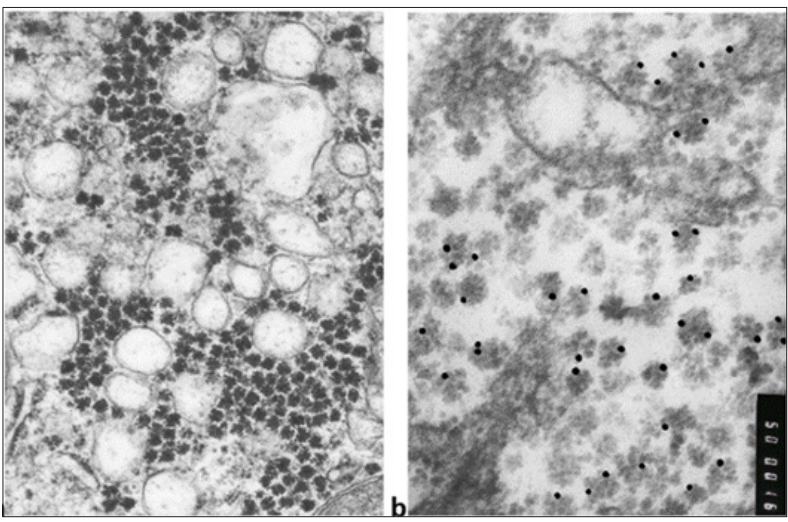

Figure 2: (a) Multiple HBV -virions in the cytoplasm of hepatocytes patient OHB. x 20 000;

(b) The product IHC reaction with monoclonal antibodies to HBsAg and complex Protein A Colloidal Gold $15 \mathrm{~nm}$ on virions. The slices is not contrasted. x 50000 .

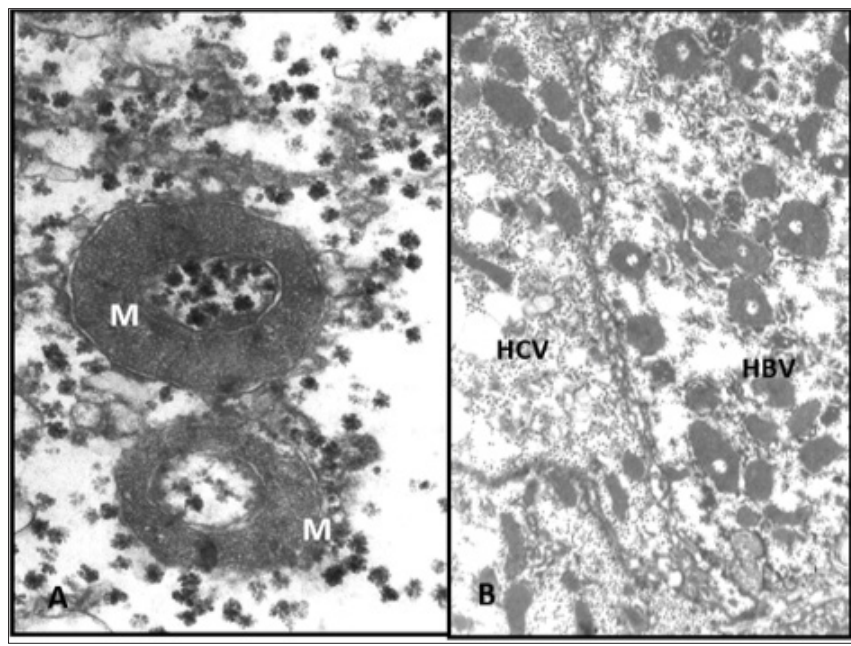

Figure 3: Specific to HGW toroidal mitochondria.

(a) The subtle structure of the mitochondria (explanations in the text). X 30,000;

(b) Multiple toroidal mitochondria in the cytoplasm of hepatocyte with HBV-virions and their absence in hepatocyte with HCV-virions in mixt infection HBV and HCV. $\mathrm{M}$ - mitochondria. $\mathrm{x}$ 6,000 .

The fact of the presence of virions not only confirms the results of ICC verification of latent HBV infection, but also proves the viral nature of diseases, and also refutes the initial clinical diagnosis. In the accumulation phase, the cytoplasm of most hepatocytes is densely filled with HBV virions. Activation of the lysosomal apparatus and active phagocytosis of viruses are noted. As a result, a large number of residual bodies are formed containing lipofuscin and myelin-like lamellae. Para crystalline protein formations are often observed in the cytoplasm and in the matrix of mitochondria. Most mitochondria have a deenergized appearance with a small number of cristae and a medium density matrix. However, among them there are very large, elongated organelles with an unusually large number of longitudinally spaced cristae and an enlightened matrix. Such an unusual hypertrophy of the inner membrane without a corresponding enhancement of function may result from a mutation of mitochondrial DNA (due to the expansion of viral DNA nucleotides) and the synthesis of altered structural proteins. The above changes are nonspecific, as they occur with other viral hepatitis. However, when monoinfection HBV we discovered annular (toroidal) mitochondria with moderate density matrix (Figure 3a \& 3b) and a large length of the inner membrane, which is not observed in other viral hepatitis. Such changes in the mitochondria, along with the presence of HBV-virion are, in our view, specific features chronic course of viral hepatitis.

Changes in the biochemical parameters of inflammation in the examined patients varied within such wide limits that it was not possible to assess additional markers of transferred HBV infection during chronic liver disease. However, it should be noted that the level of alanine and aspartic aminotransferase (ALT, AST) during coinfection HBV to HCV was significantly higher than when monoinfection HBV. During co-infection, the ALT level varied in the range of 39-406IU/L (average-120.85 $\pm 26.7 \mathrm{IU} / \mathrm{L}$ ), and AST -25139IU/L (average $-88.4 \pm 12.8 \mathrm{IU} / \mathrm{L}$ ), while monoinfection of HBV ALT averaged 49.6 $\pm 6.3 \mathrm{IU} / \mathrm{L}$, and AST-57.1 $\pm 7.5 \mathrm{IU} / \mathrm{L}$. The cases of latent HBV monoinfection that we studied were characterized by minimal clinical, biochemical and morphological changes, as well as a low level of viremia and the absence of amino acid substitutions in the structure of HBsAg that could affect the efficiency of its detection in ELISA. The presence of only stably detectable antiHBcore IgG is usually considered as evidence of a previous infection with elimination of the virus and disease remission. All patients with anti-H Bcore were found to have HBV in their liver tissue, including all patients with chronic hepatitis C. In our studies, anti -HBc was re-determined to eliminate false-positive results. On this basis, it can be argued that the presence of anti -HBc serum testifies not transferred HBV -infection, and the presence of HBV -virions in liver tissue, and that anti - HBc can be an effective, , according to experts, a marker om HBV infection. The absence of a detectable level of HBV DNA in blood serum is not a fact that excludes the presence of a latent infection. In such cases, the virus may be located in the liver tissue. This phenomenon is based on the mechanism of replication of this virus itself: the nucleus of an infected hepatocyte may contain a sufficient number of copies of ccc DNA, a stable matrix for transcription of viral proteins and replication of virions [26].

In our opinion, coinfection with HBV with various hepatotropic and non-hepatotropic viruses can be smiling the most significant cause of latent forms of chronic hepatitis, because the frequency of chronic mixt hepatitis caused by several hepatotropic viruses, has increased significantly in recent years. Even we conducted the study, all patients with chronic hepatitis $\mathrm{C}$, as well as patient with hepatitis caused by TTV, were co-infected with HBV. The most frequent co-infections include Anelloviruses, the prevalence of which approaches $100 \%$ not only in humans, but also in most mammals [27]. They exhibit properties as a parenterally 
transmitted virus, and enteral, and can be transmitted all the ways, except for the aerosol-droplets. Because viruses of this group have multiple genotypes, each of which has a tropism to specific tissues and organs in the human body can exist multiple genotypes of TTV , TT midi virus (TTMDV) and TT -like mini virus (TTMV) , although not all of them cause pathologists the liver. TTMV detected in average, 8 of 10 patients with chronic liver disease in lymphocytes, neutrophils and platelets liver blood sinusoids. Recently, however, we have succeeded in 6 patients with chronic hepatitis B (y2/6with occult HBV) identified numerous virions TTMV, located in the nuclei of hepatocytes, which proves their hepatotropic.

Thus, our study showed that the inclusion in the evaluation of patients for determining the of anti -HBcore serum changes the understanding of the etiology of chronic liver disease. It was found that the occurrence of latent form HBV among patients in the gastroenterological department is extremely high and amounts to at least $20 \%$. The results are consistent with published data. For example, in a recent study Castillo et al. [28], determined HBV DNA and HCV RNA in liver tissue in 76 HBsAg-negative patients with a stable increase in ALT activity observed over 2 years. In this group, covert HBV infection was detected in $22 \%$ of patients, HCV infection in $46 \%$, coinfection of both viruses in $32 \%$ (i.e., HBV DNA and HCV RNA were detected in liver tissue in $54 \%$ of patients). We can conclude that on the basis of the results LHB -a form of chronic HBV -infection, the difficulty of diagnosis Which a second largely exaggerated and is due, primarily, disadvantages of diagnostic standards and methodical equipment of municipal hospitals. Just introducing the definition of anti - HBc into the diagnostic standards will make it possible to reliably detect the presence of HBV infection in a patient.

\section{References}

1. Hollinger FB (2008) Hepatitis B virus infection and transfusion medicine: science and the occult. Transfusion 48(5): 1001-1026.

2. Raimondo G, Jean PA, Maurizia RB, Marie AB, Ding SC, et al. (2008) Statements from the taormina expert meeting on occult hepatitis $B$ virus infection. J Hepatol 49(4): 652-657.

3. Hollinger FB, Sood G (2010) Occult hepatitis B virus infection: A covert operation. J Viral Hepat 17(1): 1-15.

4. Torbenson M, Thomas DL (2002) Occult hepatitis B. Lancet Infect Dis 2(8): 479-486.

5. McMahon BJ, Holck P, Bulkow L, Snowball M (2001) Serologic and clinical outcomes of 1536 Alaska natives chronically infected with hepatitis B virus. Ann Intern Med 135(9): 759-768.

6. Zerbini A, Pilli M, Boni C, Fisicaro P, Penna A, et al. (2008) The characteristics of the cell-mediated immune response identify different profiles of occult hepatitis B virus infection. Gastroenterol 134(5): 14701481

7. Hu KQ (2002) Occult hepatitis B virus infection and its clinical implications. J Viral Hepat 9(4): 243-257.

8. Weinberger KM, Bauer T, Bohm S, Jilg WG (2000) High genetic variability of the group-specific a-determinant of hepatitis B virus surface antigen (HBsAg) and the corresponding fragment of the viral polymerase in chronic virus carriers lacking detectable HBsAg in serum. J Gen Virol 81(5): 1165-1174.
9. Ohto H, Ujiie N, Takeuchi C, Sato A, Hayashi A, et al. (2002) TT Virus Infection During Childhood. Transfusion 42(7): 892-898.

10. Mimms LT, Mosley JW, Hollinger FB, Aach RD, Stevens CE, et al. (1993) Effect of concurrent acute infection with hepatitis $\mathrm{C}$ virus on acute hepatitis B virus infection. BMJ 307(6912): 1095-1097.

11. Pasquinelli C, Shoenberger JM, Chung J, Chang KM, Guidotti LG, et al. (1997) Hepatitis C virus core and E2 protein expression in transgenic mice. J Hepatol 25(3): 719-727.

12. Bellecave R, Gouttenoire J, Gajer M, Brass V, Koutsoudakis G, et al. (2009) Hepatitis $B$ and $C$ virus coinfection: A novel model system reveals the absence of direct viral interference. J Hepatol 50(1): 46-55.

13. Chu CJ, Lee SD (2008) Hepatitis B virus/hepatitis C virus coinfection: Epidemiology, clinical features, viral interactions and treatment. J Gastroenterol Hepatol 23(4): 512-520.

14. Carman WF (1997) The clinical significance of surface antigen variants of hepatitis B virus. J Viral Hepat 4 Suppl 1: 11-20.

15. Fang Y, Shang QL, Liu JY, Di Li, Wei ZX, et al. (2009) Prevalence of occult hepatitis B virus infection among hepatopathy patients and healthy people in China. J Infect 58(5): 383-388.

16. Cacciola I, Pollicino T, Squadrito G, Cerenzia G, Orlando ME, et al. (1999) Occult hepatitis $B$ virus infection in patients with chronic hepatitis $C$ liver disease. N Engl J Med 341(1): 22-26.

17. Raimondo G, Navarra G, Mondello S, Costantino, Colloredo G, et al. (2008) Occult hepatitis B virus in liver tissue of individuals without hepatic disease. J Hepatol 48(5): 743-746.

18. Marusawa H, Uemoto S, Hijikata M, Y Ueda, K Tanaka, (2000) Latent hepatitis $\mathrm{B}$ virus infection in healthy individuals with antibodies to hepatitis B core antigen. J Hepatol 31(2): 488-495.

19. Coffin CS, Michalak TI (1999) Persistence of infectious hepadnavirus in the offspring of woodchuck mothers recovered from viral hepatitis. J Clin Invest 104(2): 203-212.

20. Coffin CS, Pham TNQ, Mulrooney PM, Norma D, Tomasz IM (2004) Persistence of isolated antibodies to woodchuck hepatitis virus core antigen is indicative of occult infection. Hepatol 40(5): 1053-1061.

21. Michalak TI, Pasquinelli C, Guilhot S, Chisari FV (1994) Hepatitis B virus persistence after recovery from acute viral hepatitis. J Clin Invest 93(1): 230-239.

22. Rajender Reddy K, Beavers KL, Hammond SP, Lim JK, Yngve TFY, et al. (2015) American gastroenterological association institute guideline on the prevention and treatment of hepatitis $b$ virus reactivation during immunosuppressive drug therapy. Gastroenterology 148(1): 215-219.

23. Matcha BSJ, Cao K, Jason M, Ajao A, Jones SC, et al. (2017) Hepatitis B virus reactivation associated with direct-acting antiviral therapy for chronic hepatitis c virus a review of cases reported to the U.S. food and drug administration adverse event reporting system. Ann Intern Med 166(11): 792-798.

24. Madejon A, Manzano ML, Arocena C, I Castillo, V Carreno (2000) Effects of delayed freezing of liver biopsies on the detection of hepatitis $\mathrm{C}$ virus RNA strands. J Hepatol 32(6): 1019-1025.

25. Morozov IA, Yu IL, Kuregyan KK (2011) Latent HBV infection and its detection algorithm in patients with chronic liver diseases. In the world of viral hepatitis 1: 19-26.

26. Jeantet D, Chemin I, Mandrand B, Albert T, Fabien Z et al. (2004) Cloning and expression of surface antigens from occult chronic hepatitis B virus infections and their recognition by commercial detection assays. J Med Virol 73(4): 508-515.

27. Ninomiya M, Takahashi M, Nishizawa T, Shimosegawa T, Okamoto H (2008) Development of PCR assays with nested primers specific 
for differential detection of the three human anelloviruses and early acquisition of dual or triple infection during infancy. J Clin Microbiol 46(2): 507-514.

28. Castillo I, Ilcigo RE, Alcorocho JL, Alcorocho JML, Bartolomé J, et al. (2007) Comparative study on the clinical and virological characteristics among patients with single occult hepatitis B virus (HBV), single occult hepatitis $\mathrm{C}$ virus (HCV) and occult HBV and HCV dual infection. J Med Virol 79(3): 236-241.

For possible submissions Click below:

\section{Submit Article}

